\title{
Rehabilitation, Physical and Rehabilitation Medicine and Community Based Rehabilitation - a comment to the debate towards a differentiated view of PRM on rehabilitation system, services and training of rehabilitation professionals
}

\section{Christoph Gutenbrunner, Boya Nugraha}

\section{Contributions:}

CG contributed in conception and designing framework. All authors participated in drafting and revising for intellectual content and gave final approval for submission.

\begin{abstract}
This comment aims to give a contribution to the debate about the best way to implement rehabilitation services and, in particular, how specialist in Physical and Rehabilitation Medicine (PRM) define its role in rehabilitation services in general and in Community Based Rehabilitation (CBR). The paper discusses the use of the team rehabilitation and the definitions of CBR. A differentiated model of rehabilitation services according to the phase of the disease and the intensity of service provision is developed. The term rehabilitation nowadays is used in two different ways: Rehabilitation as a health strategy or a set of measures. The term CBR also is used in a dual way on the one hand describing a policy or management strategy or on the other hand describing the provision of "basic" rehabilitation services which is offered at the community level. It is important to differentiate between acute, post-acute rehabilitation and long-term rehabilitation services to understand the need of different types of rehabilitation services. Additionally a 5-level model of the intensity of rehabilitation services covers rehabilitation that is delivered by families, peers, neighbours, and others to highly specialized rehabilitation services, e.g. for acute rehabilitation or rehabilitation for severely affected patients. In comprehensive rehabilitation service, including CBR, the role of PRM specialists is described in three ways: PRM deliver rehabilitation services, PRM act as an advisor and coordinator or PRM act as a trainer.
\end{abstract}

Keywords: Rehabilitation definition, community based rehabilitation, rehabilitation services, Physical and Rehabilitation Medicine

\section{Correspondence Details:}

Prof. Dr. med. Christoph Gutenbrunner, MD

Dept. Rehabilitation Medicine, Hannover Medical School Carl Neuberg Str. 1

30625 Hannover

Germany

Tel: +49-511-532-4100

Fax: +49-511-532-4293

Email: Gutenbrunner.Christoph@mh-hannover.de

\section{Boya Nugraha, MS, PhD}

Dept. Rehabilitation Medicine, Hannover Medical School Carl Neuberg Str. 1

30625 Hannover

Germany

Email: boya.nugraha@gmail.com 


\section{INTRODUCTION}

Since many years it has been discussed who is most qualified to deliver rehabilitation services to people with disabilities or chronic disabling health conditions. Is it the specialists for Physical and Rehabilitation Medicine (PRM), another medical specialist, a family doctor, a physiotherapist, an occupational therapist, social worker or any other person? Many papers show, that the outcomes are best if a team of different rehabilitation professional deliver the services. However, what has to be done if such a team is not available (e.g. in lower income countries?) Obviously there is not a simple answer to these questions. In particular, this is true for the discussion about Community Based rehabilitation (CBR). Here the spectrum of recommendations ranges from education of non-professionals to deliver some rehabilitation to the establishment of a PRM specialist to reach all population.

This comment will give a contribution to this kind of debate and argue from different views. This briefly discusses the use of the terms of rehabilitation in general and of CBR. It includes a differentiation of rehabilitation services along the phase of disease and the level of intensity. Last but not least it contains a proposal how to describe the role of PRM-specialists on CBR. However, the authors do not claim to give final or definite solution, as the purposed to contribute to an important and open debate.

\section{DISCUSSION}

\section{Definitions of Rehabilitation}

Rehabilitation is often seen as some kind of aftercare (e.g. after acute disease or trauma) or as physical intervention such as physiotherapy or training (e.g. after sports injury or in musculoskeletal disorders). However, in the last decades the term rehabilitation has been discussed and defined in a much more comprehensive way.

The new definitions and descriptions have two main directions:

1. Rehabilitation as a "health strategy which, based on WHO's integrative model of functioning, disability and health applies and integrates approaches to assess functioning (...), approaches to optimize a person's capacity, (...) strengthen the resources of the person, (...) and that provide a facilitating environment, (...) with the goal to enable persons with health conditions experiencing or likely to experience disability to achieve and maintain optimal functioning in interaction with their environment"

2. Rehabilitation as "a set of measures that assist individuals who experience, or are likely to experience, disability to achieve and maintain optimal functioning in interaction with their environments"

The first definition was a result of consensus process within European and International PRM societies. The second definition was referred to the WHO in the World Report on Disability. Of course other definition can be found in literature, however, in principle other definitions refer to the above concepts. Additionally, both approaches are compatible in defining optimal functioning of people with disabilities as main goal of rehabilitation. Although, the first approach shows some differences: the description of rehabilitation as a health strategy classifies rehabilitation in the framework of other health strategies, such as the curative, the preventive and the supportive strategies. It also 
includes other sectors such as education, labour and social affairs. ${ }^{1,3}$ The second description of rehabilitation as a set of measures is more focussed on medical interventions and addressed to the person with disability him or herself.

\section{Definition of Community Based}

\section{Rehabilitation}

There is no doubt that comprehensive definitions and descriptions are very important especially to build up rehabilitation systems and to benchmark them at an international level. However, it remains a problem to apply these definitions to concrete rehabilitation services or programs and even can lead to some confusion in using rehabilitation related terms. One example for this is the use of the term "Community Based Rehabilitation" $(C B R)$ (figure 1): the term of
CBR is used in two ways:

A. the first one is the policy or management strategy of CBR that was developed by WHO about 30 years ago. ${ }^{4}$ This strategy aims to strengthen the access to primary care and to specialized rehabilitation services, which is depending on the patient's needs as well as to remove barriers from the environment that hinder persons with disability in full participation in all life areas.

B. the second one is the provision of "basic" rehabilitation services which is offered at the community level. Such services may be part of the primary care system or organised as independent programs in case of absence of the first.

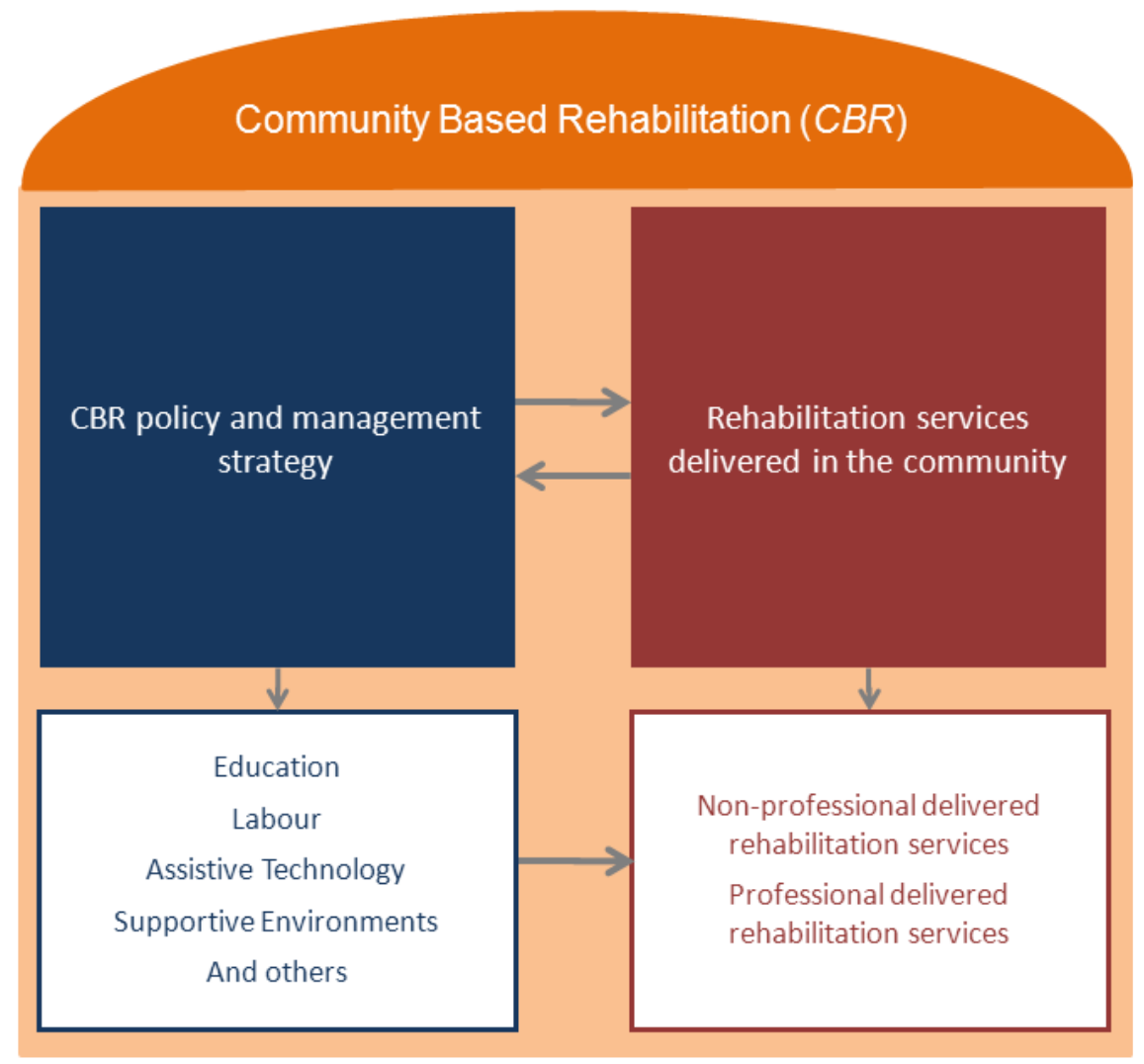

Figure 1. Community Based Rehabilitation as a policy and management strategy and as rehabilitation service delivery 
Recently, a discussion occurred whether CBR means that rehabilitation services mainly should be delivered by non-professionals or by primary care health professionals such as primary care physicians, nurses or physiotherapists, or if rehabilitation specialists must be responsible for such services. However, focusing on rehabilitation services is of major importance to understand that rehabilitation services and programs must be focussed on the (specific) needs of persons with disabilities. This among others - must reflect the phase of the disease and the level of intensity (including complexity) of interventions as well as the level of specialisation of the service providers.

\section{Phases and levels of rehabilitation services}

The phases of rehabilitation can roughly classified by the WHO's model of acute, postacute and long-term care ${ }^{2,5-7}$ :

- Acute rehabilitation services mostly are delivered in or nearby to (acute) hospitals. Acute rehabilitation services may be delivered on specialised wards (Acute rehabilitation units, $A R U$ ) or in mobile (peripatetic) acute rehabilitation teams $(M R T)$. Acute rehabilitation ensures early rehabilitation services already during or immediately after intensive care (even in patients with mechanical ventilation $)^{8}$

- Post-acute rehabilitation services rehabilitate patients after discharge from hospitals aiming at independent living and/or return-to work of patients after severe disease or injury. They are mostly led by PRM or other medical specialists. Post-acute rehabilitation services mostly are specialised in specific health conditions (e.g. spinal cord Injury, cancer, neurological or musculoskeletal conditions, or others $)^{9}$

- $\quad$ Long-term rehabilitation services aim at long-term care for persons with chronic disabilities and/or chronic health conditions. Services may vary significantly and may range from

- Non-professional delivered rehabilitation services in the community (see below)

- Rehabilitation within primary care

- Outpatient rehabilitations services delivered by rehabilitation professionals such as PRM doctors

- Intermittent in-patient or day-clinic rehabilitation services for patients with chronic health conditions (such services are developed in particular in Central Europe)

The level of specialisation of rehabilitation service providers has not been classified yet in a comprehensive way. It seems to be appropriate to use a five-level-model (figure 2). These five levels are:

- $\quad$ Level 1: Families, peers, neighbours, and others. Services at this level are mainly delivered by non-professionals, however, with time they achieve a certain level of expertise in the specific needs of the person

- Level 2: Primary care physicians, physiotherapists, occupational therapists and other general health professionals. With regard to professional training these providers are health professionals but do not have specific training in rehabilitation

- Level 3: Physical and Rehabilitation Medicine specialists, specialized Physiotherapists, Occupational Therapists and other specialised rehabilitation professionals. These health professionals have a specific postgraduate training in rehabilitation in addition to the basic professional education

- Level 4: Multi-professional rehabilitation services. These services are delivered 
by multi-professional teams of health professionals as described at level 3, team work must be structured- and patientcentred $^{10}$

- $\quad$ Level 5: Highly specialized rehabilitation services (such as tertiary level) e.g. for acute rehabilitation or rehabilitation for severely affected patients with traumatic brain injury, spinal cord injury, multiple trauma, limb amputation organ transplantation or other diseases. The service is delivered by multi-professional teams as described at level 4 that have specific knowledge in the respective disease.

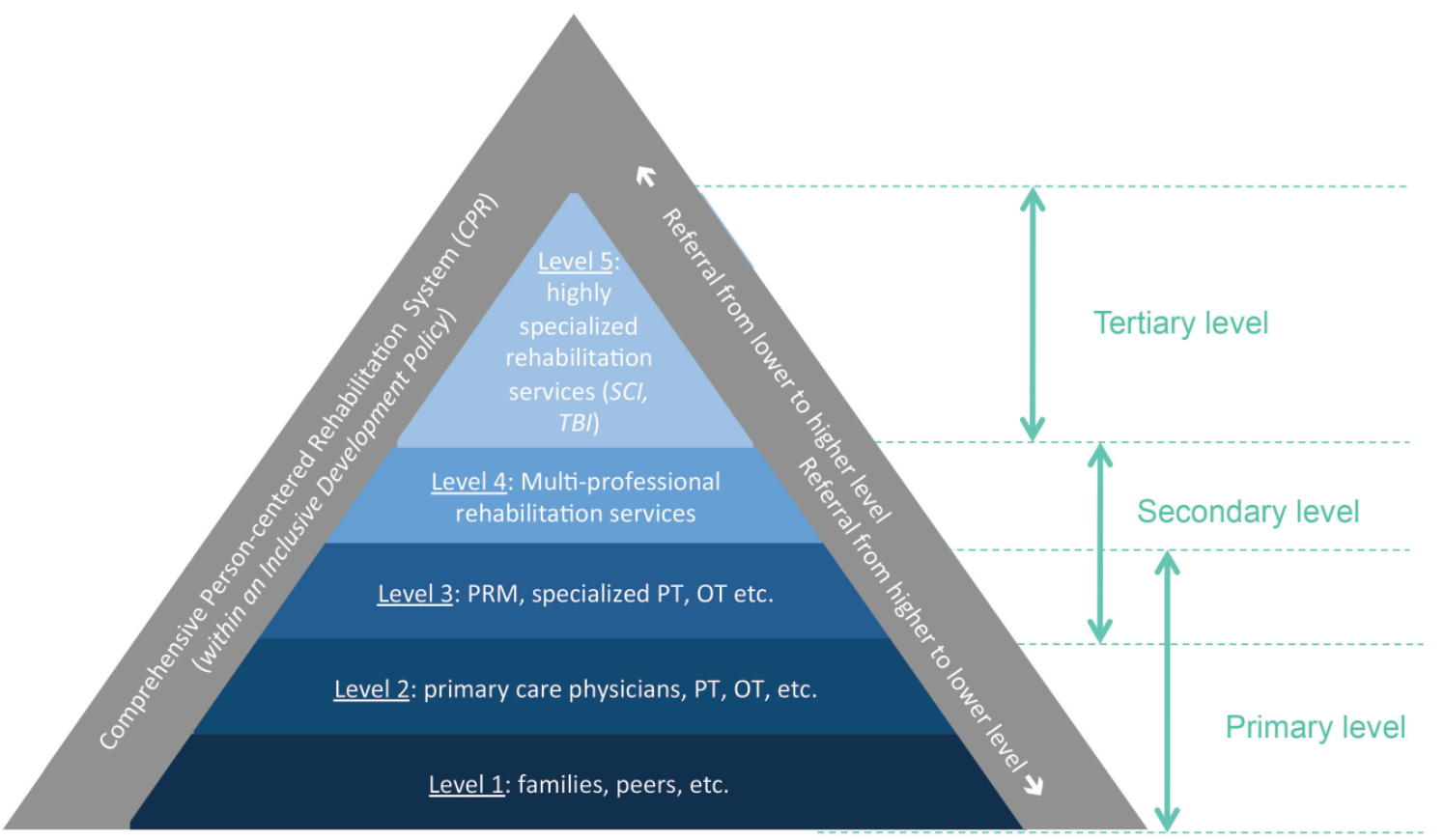

Figure 2. Rehabilitation care pyramid (an Illustration of the five levels of rehabilitation care) ${ }^{11}$

As many patients or persons with disabilities will need services of different specialisation (either consecutively or in parallel) a coordination of these services is of major importance. This must include case management and clear criteria for referral as well as an agreement on treatment and intervention concepts.

The above described conceptual approach also avoids a discussion if CBR should be delivered either by non-professionals or by trained rehabilitation professionals. It makes clear that the optimal way to organize rehabilitation is that it is delivered by non-professionals as well as by trained rehabilitation professionals. Within such a framework the choice of the right service is dependent by many factors. Two of the main factors are:

- The need of a person with a health condition experiencing disability for medical and rehabilitation care. It is obvious that a patient with spinal cord injury or in the early phase after multiple trauma needs a more specialised rehabilitation care as a person with deficits in self-care because of frailty in long-term care. However, a transfer between rehabilitation services of different levels will be necessary in many cases (e.g. transfer of patients with limb loss after fitting the prosthesis into community life) 
- The availability of rehabilitation professionals. $^{2}$ If they are not available or do not have enough capacity, service provider may have to compensate the lack of specialised rehabilitation even though the optimal level of intervention may not be reached. However, in such situation governments and stakeholders are responsible to develop the rehabilitation system of the county or region.

Of course technical and financial resources as well as accessibility are other important factors, too.

\section{CONCLUSION}

The possible role of Physical and Rehabilitation Medicine in CBR

In such a concept, PRM specialists may take different roles at different levels and phases of service delivery: ${ }^{5,6}$

- $\quad$ PRM delivers rehabilitation services: This is the core business of PRM doctors as described in the White Books of Physical and Rehabilitation Medicine in Europe ${ }^{5}$ and in Latin America ${ }^{11}$ as well as in many papers describing the field of competence of PRM. ${ }^{3,5-7,11-16}$

- $\quad$ PRM acts as an advisor and coordinator: It is crucial that rehabilitation services work in a homogenous way and use synergies, especially as transfers between the levels will occur frequently (see above). Thus, it is important to develop standards and pathways for rehabilitation care across levels (and across sectors, too). ${ }^{3}$ Additionally, referral must be managed in an appropriate and efficient way. Also here, the decision making needs a lot of background knowledge and ability to assess the patient's functional status and need for interventions. PRM has the skills for both tasks., 5

- $\quad$ PRM acts as a trainer: There is no doubt that non-professionals delivering rehabilitation care need some training and advice. The same case is for primary care health professionals who in most cases have not been educated in rehabilitation medicine at the level of the under- and postgraduate training. ${ }^{5,13,14}$ Within this context, PRM can deliver training courses at all levels of education: undergraduate, postgraduate and continuous medical education/continuous professional development.

In most national curricula of PRM all three areas are covered, however, assurance is needed to approve that rehabilitation medicine specialists are empowered to act as advisor and coordinator as well as trainer, especially if they are going to work in low-income countries where the infrastructure is still suboptimal.

All in all, it can be seen that rehabilitation services include a great variety of different settings, goals and therefore need different levels of specialisation. Moreover, it is obvious that the term rehabilitation in different contexts is used in different ways, and this is in particular the case in CBR. Thus, it is necessary to define and describe rehabilitation services, e.g. by using a classification system ${ }^{16,17}$ that at least define the service provider (including location, organisation, human and technical resources, service organisation and others), the funding of the service (describing the main sources of income and refunding of services), the service delivery (including the main strategy applied to the users, aspects of intensity and specialisation, the duration of intervention 
and the way the service is organised, e.g. team structure). ${ }^{16}$ Additionally standards and pathways for rehabilitation service delivery should be developed based on a conceptual framework, the need of rehabilitation and the potential for rehabilitation delivery that will vary from region to region. ${ }^{2}$

\section{REFERENCES}

1. Meyer T, Gutenbrunner C, Bickenbach J, Cieza A, Melvin J, Stucki G: Towards a conceptual description of rehabilitation as health strategy. J Rehabil Med 2011; 43: 765-9

2. World Heath Organisation \& World Bank: World Report on Disability. Geneva \& New York, June 2011

3. Gutenbrunner C, Meyer T, Stucki G: The Field of Competence in Physical and Rehabilitation Medicine in Light of Health Classifications - an International Perspective (Invited Commentary): Am J Phys Med Rehabil 2011a; 90: 521-25

4. World Heath Organisation, ILO, UNESCO, IDDC: Community-based rehabilitation: CBR guidelines. Introductory Booklet. Malta 2010

5. Gutenbrunner $\mathrm{C}$, Ward $\mathrm{AB}$, Chamberlain MA: White Book on Physical and Rehabilitation Medicine in Europe. Eura Medicophys 2006; 42: 287-332; Eur J Phys Rehabil Med 2009; 47 (Suppl. 1): 1-46

6. Gutenbrunner $\mathrm{C}$, Ward $\mathrm{AB}$, Chamberlain MA: White Book on Physical and Rehabilitation Medicine in Europe. J Rehabil Med 2007; 45, Suppl. 1: 1-48

7. Gutenbrunner C, Meyer T, Melvin J, Stucki G: Towards a conceptual description of Physical and Rehabilitation Medicine. J Rehabil Med 2011b; 43: 760-4
8. Ward AB, Gutenbrunner C, Damjan H, ] Giustini A, Delarque A: European Union of Medical Specialists (UEMS) section of Physical \& Rehabilitation Medicine: A Position Paper on Physical and Rehabilitation Medicine in Acute Settings. J Rehabil Med 2010, 42: 417-24

9. Ward AB, Gutenbrunner C, Giustini A, A, Fialka-Moser V, Kiekens C, Berteanu, M, Christodoulou: European Union of Medical Specialists (UEMS) Section of Physical \& Rehabilitation Medicine: A Position Paper on Physical and Rehabilitation Medicine in Post-Acute Settings. J Rehabil Med 2012; 44: 289-98

10. Neumann V, Gutenbrunner C, Fialka-Moser V, Christodoulou N, Varela E, Giustini A, Delarque A: Interdisciplinary Team Working in Physical and Rehabilitation Medicine. J Rehab Med 2010; 42: 4-8

11. Gutenbrunner $\mathrm{C}$ and Nugraha B. Physical and rehabilitation medicine: responding to health needs from individual care to service provision. Eur J Phys Rehabil Med. 2017 Feb;53(1):1-6

12. Gutenbrunner $\mathrm{C}$, Abuchaibe S, Lugo LH, Escobar LM (Eds): Libro Blanco de Medicina Física y Rehabilitatión en América Latina. Idenografic SAS, Medellin, Columbia 2012

13. Gutenbrunner C, Neumann V, Lemoine F, Delarque A: Describing and developing the field of competence in Physical and Rehabilitation Medicine (PRM) in Europe - preface to a series of papers published by the Professional Practice Committee of the PRM section of the Union of European Medical Specialists (UEMS)/ Décrire et développer le champ de compétences en Médecine Physique et Réadaptation (MPR) en Europe. Préface d'une série d'articles publiés par le Comité des 
pratiques professionnelles de la sec-tion MPR de l'Union européenne des médecins spécialistes (UEMS)(Editorial/Éditorial). Ann Phys Rehab Med 2010a; 53: 593-7

14. Gutenbrunner C, Schiller J, Schwarze M, Fischer V, Paulmann V, Haller H, Küther G: The Hannover Model for the Implementation of Physical and Rehabilitation Medicine into Undergraduate Medical Training. J Rehab Med 2010b;42:206-13

15. Gutenbrunner $\mathrm{C}$, Lemoine $\mathrm{F}$, Yelnik A, Joseph PA, de Korvin G, Neumann V, Delarque A: The field of competence of the specialist in physical and rehabilitation medicine (PRM) - Champ de compétence du spécialiste en médecine physique et de réadaptation. Ann Phys Rehabil Med 2011c; 54: 298-318

16. Gutenbrunner C, Bickenbach J, Kiekens C, Meyer T, Skempes D, Nugraha B, Bethge M, Stucki G: ISPRM Discussion Paper: Proposing Dimensions for an International Classification System for Service Organisation in Health-related Rehabilitation (ICSO-R). J Rehabil Med. 2015 Oct 5;47(9):809-15

17. Meyer T, Gutenbrunner C, Kiekens C, Skempes D, Melvin JL, Schedler K, Imamura M, Stucki G: ISPRM discussion paper: Proposing a conceptual description of health-related rehabilitation services. J Rehabil Med 2014; 46: 1-6 